\title{
A generalized $q$-fractional Gronwall inequality and its applications to nonlinear delay $q$-fractional difference systems
}

\author{
Thabet Abdeljawad ${ }^{1 *}$, Jehad Alzabut ${ }^{1}$ and Dumitru Baleanu ${ }^{2,3}$
}

\author{
${ }^{*}$ Correspondence: \\ tabdeljawad@psu.edu.sa \\ 1 Department of Mathematics and \\ Physical Sciences, Prince Sultan \\ University P. O. Box 66833, Riyadh, \\ 11586, Saudi Arabia \\ Full list of author information is \\ available at the end of the article
}

\begin{abstract}
In this paper, we state and prove a new discrete $q$-fractional version of the Gronwall inequality. Based on this result, a particular version expressed by means of the q-Mittag-Leffler function is provided. To apply the proposed results, we prove the uniqueness and obtain an estimate for the solutions of nonlinear delay Caputo $q$-fractional difference system. We examine our results by providing a numerical example.
\end{abstract}

MSC: $26 \mathrm{~A} 33 ; 39 \mathrm{~A} 11$

Keywords: generalized $q$-fractional Gronwall inequality; delay $q$-fractional difference system; uniqueness of solution; estimate for the solution

\section{Background}

The study of $q$-difference equations has gained intensive interest in the last years. It has been shown that these types of equations have numerous applications in diverse fields and thus have evolved into multidisciplinary subjects [1-10]. For more details on $q$-calculus, we refer the reader to the remarkable monograph [11]. On the other hand, the fractional differential equations have recently received considerable attention in the last two decades. Indeed, many researchers have investigated these types of equations due to their significant applications in various fields of science and engineering; see for instance the monographs [12-14] and the references therein.

The corresponding theory of fractional difference equations is considered to be at its first stages of progress; we suggest [15-26] whose authors have taken the lead to promote and develop this theory. The $q$-fractional calculus and differential equations have been recently studied in many papers; we recommend the monograph [27] and the papers cited therein. For the $q$-fractional difference equations which serve as a bridge between fractional difference equations and $q$-difference equations there have appeared some papers which study the qualitative properties of solutions [15, 27-33]. However, less attention has been paid to these types of equations in the literature.

The differential and integral inequalities, which are considered as an effective tools for studying solutions properties, have also been under consideration. Due to its benefit in the determination of uniqueness, boundedness and stability of solutions, in particular, the

(c) 2016 Abdeljawad et al. This article is distributed under the terms of the Creative Commons Attribution 4.0 International License (http://creativecommons.org/licenses/by/4.0/), which permits unrestricted use, distribution, and reproduction in any medium, provided you give appropriate credit to the original author(s) and the source, provide a link to the Creative Commons license, and indicate if changes were made. 
Gronwall inequality has been a main target for many researchers. There have appeared several versions for the Gronwall inequality in the literature; we list here those results which concern with fractional differential or difference equations [34-39].

For $0<q<1$, we define the time scale $\mathbb{T}_{q}=\left\{q^{n}: n \in \mathbb{Z}\right\} \cup\{0\}$, where $\mathbb{Z}$ is the set of integers. For $a=q^{n_{0}}$ and $n_{0} \in \mathbb{Z}$, we denote $\mathbb{T}_{a}=[a, \infty)_{q}=\left\{q^{-i} a: i=0,1,2, \ldots\right\}$. In [40], which is probably the first paper in this subject, the current authors have established a discrete $q$-fractional version of the Gronwall inequality. Indeed, they obtained the theorem given below.

Theorem 1 [40] Let $\alpha>0, u$ and $\mu$ be nonnegative real valued functions such that $0 \leq$ $\mu(t)<\frac{1}{t^{\alpha}(1-q)^{\alpha}}$ for all $t \in \mathbb{T}_{a}$ (in particular if $\left.0 \leq \mu(t)<\frac{1}{(1-q)^{\alpha}}\right)$ and

$$
u(t) \leq u(a)+{ }_{q} \nabla_{a}^{-\alpha} u(t) \mu(t) .
$$

Then

$$
u(t) \leq u(a) \sum_{k=0}^{\infty}{ }_{q} E_{\mu}^{k} 1
$$

where ${ }_{q} E_{\mu}^{k} 1=\frac{\mu^{k}(t-a)_{q}^{k \alpha}}{\Gamma_{q}(k \alpha+1)}$.

Based on the result of Theorem 1, the following particular estimate which is expressed by means of the $q$-Mittag-Leffler function was also concluded.

Theorem 2 [40] Let $0 \leq \delta(t)<\frac{1}{(1-q)}$ for all $t \in \mathbb{T}_{a}$. If

$$
u(t) \leq u(a)+\int_{a}^{t} \delta(s) u(s) \nabla_{q} s
$$

then

$$
u(t) \leq u(a) e_{q}(t, a)
$$

where $e_{q}(t, a)={ }_{q} E_{1}(1, t-a)$ is the nabla q-exponential function for the time scale $\mathbb{T}_{q}$.

To apply these results, the authors considered the following discrete $q$-fractional initial value problem:

$$
\left\{\begin{array}{l}
{ }_{q} C_{a}^{\alpha} x(t)=f(t, x(t)), \quad 0<\alpha \leq 1, a \in \mathbb{T}_{q}, t \in \mathbb{T}_{a}, \\
x(a)=\gamma .
\end{array}\right.
$$

Here ${ }_{q} C_{a}^{\alpha}$ means the Caputo fractional difference of order $\alpha$ and $f(t, y)$ fulfills a Lipschitz condition for all $t$ and $y$. The uniqueness of solutions as well as the dependence on the initial data were proved.

The purpose of our manuscript is to extend the results in Theorem 1 and Theorem 2 and obtain a new discrete $q$-fractional version of the Gronwall inequality valid for nonlinear systems containing delay arguments. As an application, we will prove the uniqueness and obtain an estimate for the solutions of nonlinear delay Caputo $q$-fractional difference systems. We examine our results by presenting a numerical example. 


\section{Auxiliary assertions}

Before starting, we provide some basic nabla notations, definitions, and lemmas that will be used in the sequel. Let $f: \mathbb{T}_{q} \rightarrow \mathbb{R}$. We define the nabla $q$-derivative of $f$ by

$$
\nabla_{q} f(t)=\frac{f(t)-f(q t)}{(1-q) t}, \quad t \in \mathbb{T}_{q}-\{0\}
$$

The nabla $q$-integral of $f$ has the following form:

$$
\int_{0}^{t} f(s) \nabla_{q} s=(1-q) t \sum_{i=0}^{\infty} q^{i} f\left(t q^{i}\right)
$$

and for $0 \leq a \in \mathbb{T}_{q}$

$$
\int_{a}^{t} f(s) \nabla_{q} s=\int_{0}^{t} f(s) \nabla_{q} s-\int_{0}^{a} f(s) \nabla_{q} s
$$

The definition of the $q$-factorial function for $n \in \mathbb{N}$ is given by

$$
(t-s)_{q}^{n}=\prod_{i=0}^{n-1}\left(t-q^{i} s\right) .
$$

In the case $\alpha$ is a nonpositive integer, the $q$-factorial function is defined by

$$
(t-s)_{q}^{\alpha}=t^{\alpha} \prod_{i=0}^{\infty} \frac{1-\frac{s}{t} q^{i}}{1-\frac{s}{t} q^{i+\alpha}} .
$$

Below we present some of the properties of $q$-factorial functions within the following lemma.

Lemma 1 [28] For $\alpha, \gamma, \beta \in \mathbb{R}$, we have

(i) $(t-s)_{q}^{\beta+\gamma}=(t-s)_{q}^{\beta}\left(t-q^{\beta} s\right)_{q}^{\gamma}$.

(ii) $(a t-a s)_{q}^{\beta}=a^{\beta}(t-s)_{q}^{\beta}$.

(iii) The nabla q-derivative of the q-factorial function with respect to $t$ is

$$
\nabla_{q}(t-s)_{q}^{\alpha}=\frac{1-q^{\alpha}}{1-q}(t-s)_{q}^{\alpha-1}
$$

(iv) The nabla q-derivative of the q-factorial function with respect to $s$ is

$$
\nabla_{q}(t-s)_{q}^{\alpha}=-\frac{1-q^{\alpha}}{1-q}(t-q s)_{q}^{\alpha-1}
$$

For a function $f: \mathbb{T}_{q} \rightarrow \mathbb{R}$, the left $q$-fractional integral ${ }_{q} \nabla_{a}^{-\alpha}$ of order $\alpha \neq 0,-1,-2, \ldots$ and starting at $0<a \in \mathbb{T}_{q}$ is defined by

$$
{ }_{q} \nabla_{a}^{-\alpha} f(t)=\frac{1}{\Gamma_{q}(\alpha)} \int_{a}^{t}(t-q s)_{q}^{\alpha-1} f(s) \nabla_{q} s
$$


where

$$
\Gamma_{q}(\alpha+1)=\frac{1-q^{\alpha}}{1-q} \Gamma_{q}(\alpha), \quad \Gamma_{q}(1)=1, \alpha>0 .
$$

One should note that the left $q$-fractional integral ${ }_{q} \nabla_{a}^{-\alpha}$ maps functions defined on $\mathbb{T}_{q}$ to functions defined on $\mathbb{T}_{q}$.

Definition 1 [32] Let $0<\alpha \notin \mathbb{N}$. Then the Caputo left $q$-fractional derivative of order $\alpha$ of a function $f$ defined on $\mathbb{T}_{q}$ is defined by

$$
{ }_{q} C_{a}^{\alpha} f(t) \triangleq{ }_{q} \nabla_{a}^{-(n-\alpha)} \nabla_{q}^{n} f(t)=\frac{1}{\Gamma_{q}(n-\alpha)} \int_{a}^{t}(t-q s)_{q}^{n-\alpha-1} \nabla_{q}^{n} f(s) \nabla_{q} s,
$$

where $n=[\alpha]+1$. In the case $\alpha \in \mathbb{N}$, we may write ${ }_{q} C_{a}^{\alpha} f(t) \triangleq \nabla_{q}^{n} f(t)$. The (left) Riemann $q$-fractional derivative is defined by $\left({ }_{q} \nabla_{a}^{\alpha} f\right)(t)=\left(\nabla_{q q} \nabla_{a}^{-(n-\alpha)} f\right)(t)$.

In virtue of [32], the Riemann and Caputo $q$-fractional derivatives are related by

$$
\left({ }_{q} C_{a}^{\alpha} f\right)(t)=\left({ }_{q} \nabla_{a}^{\alpha} f\right)(t)-\frac{(t-a)_{q}^{-\alpha}}{\Gamma_{q}(1-\alpha)} f(a) .
$$

Lemma 2 [32] Let $\alpha>0$ and $f$ be defined in a suitable domain. Thus

$$
{ }_{q} \nabla_{a}^{-\alpha}{ }_{q} C_{a}^{\alpha} f(t)=f(t)-\sum_{k=0}^{n-1} \frac{(t-a)_{q}^{k}}{\Gamma_{q}(k+1)} \nabla_{q}^{k} f(a)
$$

and if $0<\alpha \leq 1$ we have

$$
{ }_{q} \nabla_{a}^{-\alpha}{ }_{q} C_{a}^{\alpha} f(t)=f(t)-f(a) .
$$

The following identity plays a crucial role in solving the linear $q$-fractional equations:

$$
{ }_{q} \nabla_{a}^{-\alpha}(x-a)_{q}^{\mu}=\frac{\Gamma_{q}(\mu+1)}{\Gamma_{q}(\alpha+\mu+1)}(x-a)_{q}^{\mu+\alpha} \quad(0<a<x<b),
$$

where $\alpha \in \mathbb{R}^{+}$and $\mu \in(-1, \infty)$. The $q$-analog of the Mittag-Leffler function with double index $(\alpha, \beta)$ is introduced in [32]. It was defined as follows.

Definition 2 [32] For $z, z_{0} \in \mathbf{C}$ and $\mathfrak{R}(\alpha)>0$, the $q$-Mittag-Leffler function is defined by

$$
{ }_{q} E_{\alpha, \beta}\left(\lambda, z-z_{0}\right)=\sum_{k=0}^{\infty} \lambda^{k} \frac{\left(z-z_{0}\right)_{q}^{\alpha k}}{\Gamma_{q}(\alpha k+\beta)} .
$$

In the case $\beta=1$, we utilize ${ }_{q} E_{\alpha}\left(\lambda, z-z_{0}\right):={ }_{q} E_{\alpha, 1}\left(\lambda, z-z_{0}\right)$.

Example 1 [32] Let $0<\alpha \leq 1$ and consider the left Caputo $q$-fractional difference equation

$$
{ }_{q} C_{a}^{\alpha} y(t)=\lambda y(t)+f(t), \quad y(a)=a_{0}, t \in T_{q} .
$$


The solution of (14) is given by

$$
y(t)=a_{0 q} E_{\alpha}(\lambda, t-a)+\int_{a}^{t}(t-q s)_{q}^{\alpha-1}{ }_{q} E_{\alpha, \alpha}\left(\lambda, t-q^{\alpha} s\right) f(s) \nabla_{q} s .
$$

If instead we use the modified $q$-Mittag-Leffler function

$$
{ }_{q} e_{\alpha, \beta}\left(\lambda, z-z_{0}\right)=\sum_{k=0}^{\infty} \lambda^{k} \frac{\left(z-z_{0}\right)_{q}^{\alpha k+(\beta-1)}}{\Gamma_{q}(\alpha k+\beta)}
$$

then the solution representation becomes

$$
y(t)=a_{0 q} e_{\alpha}(\lambda, t-a)+\int_{a}^{t}{ }_{q} e_{\alpha, \alpha}(\lambda, t-q s) f(s) \nabla_{q} s .
$$

Remark 1 [32] If we set $\alpha=1, \lambda=1, a=0$, and $f(t)=0$ in (14), we obtain a $q$-exponential formula $e_{q}(t)=\sum_{k=0}^{\infty} \frac{t^{k}}{\Gamma_{q}(k+1)}$ on the time scale $T_{q}$, where $\Gamma_{q}(k+1)=[k]_{q} !=[1]_{q}[2]_{q} \cdots[k]_{q}$ with $[r]_{q}=\frac{1-q^{r}}{1-q}$. We recall that $e_{q}(t)=E_{q}((1-q) t)$, where $E_{q}(t)$ denoted a special case of the basic hypergeometric series, namely

$$
E_{q}(t)={ }_{1} \phi_{0}(0 ; q, t)=\prod_{n=0}^{\infty}\left(1-q^{n} t\right)^{-1}=\sum_{n=0}^{\infty} \frac{t^{n}}{(q)_{n}}
$$

where $(q)_{n}=(1-q)\left(1-q^{2}\right) \cdots\left(1-q^{n}\right)$ denotes the $q$-Pochhammer symbol.

\section{A generalized $q$-Gronwall inequality}

We state and prove the generalized $q$-Gronwall inequality.

Theorem 3 Let $\alpha>0, u(t), v(t)$ be nonnegative functions and $w(t)$ be nonnegative and nondecreasing function for $t \in[a, \infty)_{q}=\left\{q^{-i} a: i=0,1,2, \ldots\right\}, a=q^{n_{0}}$ for some $n_{0} \in \mathbb{Z}$ such that $w(t) \leq M$ where $M$ is a constant. If

$$
u(t) \leq v(t)+w(t)_{q} \nabla_{a}^{-\alpha} u(t)
$$

then

$$
u(t) \leq v(t)+\sum_{k=1}^{\infty}\left(w(t) \Gamma_{q}(\alpha)\right)^{k}{ }_{q} \nabla_{a}^{-k \alpha} v(t)
$$

Proof Define

$$
B \phi(t)=w(t) \int_{a}^{t}(t-q s)_{q}^{\alpha-1} \phi(s) \nabla_{q} s, \quad t \in \mathbb{T}_{a}
$$

It follows that

$$
u(t) \leq v(t)+B u(t)
$$


which implies that $u(t) \leq \sum_{k=0}^{n-1} B^{k} v(t)+B^{n} u(t)$. We claim that

$$
B^{n} u(t) \leq \int_{a}^{t} \frac{\left(w(t) \Gamma_{q}(\alpha)\right)^{n}}{\Gamma_{q}(n \alpha)}(t-q s)_{q}^{n \alpha-1} u(s) \nabla_{q} s
$$

and $B^{n} u(t) \rightarrow 0$ as $n \rightarrow \infty$ for $t \in \mathbb{T}_{a}$. It is easy to see that (17) is valid for $n=1$. Assume that it is true for $n=k$, that is,

$$
B^{k} u(t) \leq \int_{a}^{t} \frac{\left(w(t) \Gamma_{q}(\alpha)\right)^{k}}{\Gamma_{q}(k \alpha)}(t-q s)_{q}^{k \alpha-1} u(s) \nabla_{q} s
$$

If $n=k+1$, then

$$
\begin{aligned}
B^{k+1} u(t) & =B\left(B^{k} u(t)\right) \leq w^{k+1}(t) \int_{a}^{t}(t-q s)_{q}^{\alpha-1} \int_{a}^{s} \frac{\left(\Gamma_{q}(\alpha)\right)^{k}}{\Gamma_{q}(k \alpha)}(s-q r)_{q}^{k \alpha-1} u(r) \nabla_{q} r \nabla_{q} s \\
& =w^{k+1}(t) \int_{a}^{t} \int_{r}^{t} \frac{\left(\Gamma_{q}(\alpha)\right)^{k}}{\Gamma_{q}(k \alpha)}(t-q s)_{q}^{\alpha-1}(s-q r)_{q}^{k \alpha-1} u(r) \nabla_{q} s \nabla_{r} \\
& =\frac{\left(w(t) \Gamma_{q}(\alpha)\right)^{k+1}}{\Gamma_{q}(k \alpha)} \int_{a}^{t}\left[\frac{1}{\Gamma_{q}(\alpha)} \int_{r}^{t}(t-q s)_{q}^{\alpha-1}(s-q r)^{k \alpha-1} \nabla_{q} s\right] u(r) \nabla_{q} r \\
& =\frac{\left(w(t) \Gamma_{q}(\alpha)\right)^{k+1}}{\Gamma_{q}(k \alpha)} \int_{a}^{t} \nabla_{q r}^{-\alpha}(s-q r)_{q}^{k \alpha-1} u(r) \nabla_{q} r,
\end{aligned}
$$

where $\nabla_{q r}^{-\alpha} u(t)=\frac{1}{\Gamma_{q}(\alpha)} \int_{q r}^{t}(t-q s)_{q}^{\alpha-1} u(s)$ has been used. It follows from (12) that

$$
\begin{aligned}
B^{k+1} u(t) & \leq \frac{\left(w(t) \Gamma_{q}(\alpha)\right)^{k+1}}{\Gamma_{q}(k \alpha)} \int_{a}^{t}(s-q r)_{q}^{(k+1) \alpha-1} \frac{\Gamma_{q}(k \alpha)}{\Gamma_{q}((k+1) \alpha)} u(r) \nabla_{q} r \\
& =\int_{a}^{t} \frac{\left(w(t) \Gamma_{q}(\alpha)\right)^{k+1}}{\Gamma_{q}((k+1) \alpha)}(s-q r)_{q}^{(k+1) \alpha-1} u(r) \nabla_{q} r .
\end{aligned}
$$

Therefore, equation (17) is obtained. Furthermore and because the denominator goes to infinity faster than the numerator in the below inequality, one can conclude that

$$
B^{n} u(t) \leq \int_{a}^{t} \frac{\left(M \Gamma_{q}(\alpha)\right)^{n}}{\Gamma_{q}(n \alpha)}(t-q s)_{q}^{n \alpha-1} u(s) \nabla_{q} s \rightarrow 0 \quad \text { as } n \rightarrow \infty, t \in[a, \infty)_{q} .
$$

To complete the proof, we let $n \rightarrow \infty$ in

$$
u(t) \leq \sum_{k=0}^{n-1} B^{k} v(t)+B^{n} u(t)=v(t)+\sum_{k=1}^{n-1} B^{k} v(t)+B^{n} u(t)
$$

to obtain

$$
u(t) \leq v(t)+\sum_{k=1}^{\infty} B^{k} v(t)
$$

With the help of the semigroup property ${ }_{q} \nabla_{a}^{-\alpha}{ }_{q} \nabla_{a}^{-\mu}={ }_{q} \nabla_{a}^{-(\alpha+\mu)}$ and the definition of $B$ we get (16). This completes the proof. 
The following immediate consequence of the above theorem plays a key role in our subsequent analysis.

Corollary 1 Under the hypotheses of Theorem 3, assume further that $v(t)$ is a nondecreasing function for $t \in \mathbb{T}_{a}$, then

$$
u(t) \leq v(t)_{q} E_{\alpha}\left(w(t) \Gamma_{q}(\alpha), t-a\right), \quad t \in \mathbb{T}_{a} .
$$

Proof From (16) and the assumption that $v(t)$ is a nondecreasing function for $t \in \mathbb{T}_{a}$, we may write

$$
u(t) \leq v(t)\left[1+\sum_{k=1}^{\infty} \int_{a}^{t} \frac{\left(w(t) \Gamma_{q}(\alpha)\right)^{k}}{\Gamma_{q}(k \alpha)}(t-q s)_{q}^{k \alpha-1} \nabla_{q} s\right]
$$

or

$$
u(t) \leq v(t)\left[1+\sum_{k=1}^{\infty}{ }_{q} \nabla_{a}^{-k \alpha}\left(w(t) \Gamma_{q}(\alpha)\right)^{k}\right] .
$$

Then, with the help of (12) it follows that

$$
\begin{aligned}
u(t) & \leq v(t)\left[1+\sum_{k=1}^{\infty}\left(w(t) \Gamma_{q}(\alpha)\right)_{q}^{k} \nabla_{a}^{-k \alpha} 1\right] \\
& =v(t)\left[1+\sum_{k=1}^{\infty}\left(w(t) \Gamma_{q}(\alpha)\right)^{k} \frac{(t-a)_{q}^{k \alpha}}{\Gamma_{q}(k \alpha+1)}\right] \\
& =v(t) \sum_{k=0}^{\infty} \frac{\left(w(t) \Gamma_{q}(\alpha)\right)^{k}(t-a)_{q}^{k \alpha}}{\Gamma_{q}(k \alpha+1)}=v(t)_{q} E_{\alpha}\left(w(t) \Gamma_{q}(\alpha), t-a\right) .
\end{aligned}
$$

The proof is complete.

\section{Applications to nonlinear delay $q$-fractional difference systems}

Let $\mathbb{R}^{m}$ be the $m$-dimensional Euclidean space and define $\mathbb{I}_{\tau}=\left\{\tau a, q^{-1} \tau a, q^{-2} \tau a, \ldots, a\right\}$, $\mathbb{N}_{0}=\{0,1,2,3, \ldots\}$ and $\mathbb{T}_{\tau a}=[\tau a, \infty)_{q}=\left\{\tau a, q^{-1} \tau a, q^{-2} \tau a, \ldots\right\}$ where $\tau=q^{d} \in \mathbb{T}_{q}, d \in \mathbb{N}_{0}$ and $\mathbb{I}_{\tau}=\{a\}$ with $d=0$ is the non-delay case. We obtain our first application by proving the uniqueness of the solution for the system:

$$
\begin{cases}{ }_{q} C_{a}^{\alpha} x(t)=A_{0} x(t)+A_{1} x(\tau t)+f(t, x(t), x(\tau t)), & t \in[a, \infty)_{q}, \\ x(t)=\varphi(t), & t \in \mathbb{I}_{\tau},\end{cases}
$$

where ${ }_{q} C_{a}^{\alpha}$ denotes the Caputo fractional difference of order $\alpha \in(0,1)$, the state vector $x$ : $\mathbb{T}_{\tau a} \rightarrow \mathbb{R}^{m}$, the constant matrices $A_{0}$ and $A_{1}$ are of appropriate dimensions, the nonlinearity $f: \mathbb{T}_{\tau a} \times \mathbb{R}^{m} \times \mathbb{R}^{m} \rightarrow \mathbb{R}^{m}$ and the initial function $\varphi: \mathbb{I}_{\tau} \rightarrow \mathbb{R}^{m}$. Let $|\cdot|$ be any Euclidean norm and $\|\cdot\|$ be the matrix norm induced by this vector. Let $D=D\left(\mathbb{N}_{0} \times \mathbb{R}^{m} \times \mathbb{R}^{m}, \mathbb{R}^{m}\right)$ be the set of all bounded functions (sequences). Clearly, the space $D$ is a Banach space induced by the norm $\|z\|_{D}:=\sup _{t \in \mathbb{I}_{\tau}} z(t)$. 
We make use of the following assumptions:

(H.1) $f \in D\left(\mathbb{T}_{q} \times \mathbb{R}^{m} \times \mathbb{R}^{m}, \mathbb{R}^{m}\right)$ is a Lipschitz-type function. That is, there exists a positive constant $L_{1}>0$ such that

$$
\|f(t, x(t), x(\tau t))-f(t, y(t), y(\tau t))\| \leq L_{1}(\|x(t)-y(t)\|+\|x(t-\tau)-y(\tau t)\|),
$$

for $t \in[a, \infty)_{q}$.

(H.2) There exists a positive constant $L_{2}$ such that $\|f(t, x(t), x(\tau t))\| \leq L_{2}$.

The first result in this section provides a representation for the solutions of system (19) that will be useful in the subsequent analysis.

Theorem $4 x: \mathbb{T}_{\tau a} \rightarrow \mathbb{R}^{m}$ is a solution of system (19) if and only if

$$
\left\{\begin{aligned}
x(t)=\varphi(a)+ & \frac{1}{\Gamma_{q}(\alpha)} \sum_{s=1}^{t}(t-q s)_{q}^{\alpha-1}\left[A_{0} x(s)+A_{1} x(\tau s)+f(s, x(s), x(\tau s))\right] \\
& t \in[a, \infty)_{q} \\
x(t)=\varphi(t), \quad & t \in \mathbb{I}_{\tau}
\end{aligned}\right.
$$

Proof For $t \in \mathbb{I}_{\tau}$, it is clear that $x(t)=\varphi(t)$ is the solution of (19). For $t \in \mathbb{T}_{a}$, we apply ${ }_{q} \nabla_{a}^{\alpha}$ on both sides of equation (20) to obtain

$$
{ }_{q} \nabla_{a}^{\alpha} x(t)=\varphi(a) \frac{t^{-\alpha}}{\Gamma_{q}(1-\alpha)}+A_{0} x(t)+A_{1} x(\tau t)+f(t, x(t), x(\tau t)),
$$

where $\left({ }_{q} \nabla_{a q}^{\alpha} \nabla_{a}^{-\alpha} u\right)(t)=u(t)$ have been used. By using equation (9), we end up with the desired form

$$
{ }_{q} C_{t}^{\alpha} x(t)=A_{0} x(t)+A_{1} x(\tau t)+f(t, x(t), x(\tau t)), \quad t \in[a, \infty)_{q} .
$$

From system (19), we can see that $x(t)=\varphi(t)$ for $t \in \mathbb{I}_{\tau}$. For $t \in[a, \infty)_{q}$, we apply ${ }_{q} \nabla_{a}^{-\alpha}$ on both sides of equation (19) to get

$$
{ }_{q} \nabla_{a}^{-\alpha}\left[{ }_{q} C_{a}^{\alpha} x(t)\right]=\frac{1}{\Gamma_{q}(\alpha)} \int_{a}^{t}(t-q s)_{q}^{\alpha-1}\left[A_{0} x(s)+A_{1} x(\tau s)+f(s, x(s), x(\tau s)) \nabla_{q} s\right] .
$$

In view of equation (11), one can easily see that

$$
x(t)=\varphi(a)+\frac{1}{\Gamma_{q}(\alpha)} \sum_{s=1}^{t}(t-q s)_{q}^{\alpha-1}\left[A_{0} x(s)+A_{1} x(\tau s)+f(s, x(s), x(\tau s))\right] .
$$

Next we state and prove the uniqueness theorem.

Theorem 5 Let condition (H.1) hold. If $x(t)$ and $y(t)$ are two solutions for the system (19), then $x(t)=y(t)$.

Proof Let $x$ and $y$ be two solutions of system (19). Denote $z$ by $z(t)=x(t)-y(t)$. Then one can easily figure out that $z(t)=0$ for $t \in \mathbb{I}_{\tau}$. This implies that system (19) has a unique solution for $t \in \mathbb{I}_{\tau}$. 
For $t \in \mathbb{T}_{a}$, however, we have

$$
\left.z(t)=\frac{1}{\Gamma_{q}(\alpha)} \int_{a}^{t}(t-q s)_{q}\right)_{q}^{\alpha-1}\left[A_{0} z(s)+A_{1} z(\tau s)+f(s, x(s), x(\tau s))-f(s, y(s), y(\tau s))\right] \nabla_{q} s .
$$

If $t \in \mathbb{I}_{\tau^{-1}}=\left\{a, q^{-1} \tau, \ldots, \tau^{-1} a\right\}$, then $z(\tau t)=0$. Therefore,

$$
z(t)=\frac{1}{\Gamma_{q}(\alpha)} \int_{a}^{t}(t-q s)_{q}^{\alpha-1}\left[A_{0} z(s)+f(s, x(s), x(\tau s))-f(s, y(s), y(\tau s))\right] \nabla_{q} s .
$$

This implies

$$
\begin{aligned}
\|z(t)\| & \leq \frac{1}{\Gamma_{q}(\alpha)} \int_{a}^{t}(t-q s)_{q}^{\alpha-1}\left[\left\|A_{0}\right\|\|z(s)\|+\|f(s, x(s), x(\tau s))-f(s, y(s), y(\tau s))\|\right] \nabla_{q} s \\
& \leq \frac{1}{\Gamma_{q}(\alpha)} \int_{a}^{t}(t-q s)_{q}^{\alpha-1}\left[\left\|A_{0}\right\|\|z(s)\|+L_{1}(\|x(t)-y(t)\|+\|x(\tau t)-y(\tau t)\|)\right] \nabla_{q} s \\
& =\frac{1}{\Gamma_{q}(\alpha)} \int_{a}^{t}(t-q s)_{q}^{\alpha-1}\left[\left(\left\|A_{0}\right\|+L_{1}\right)\|z(s)\|+L_{1}\|z(\tau s)\|\right] \nabla_{q} s \\
& =\frac{\left\|A_{0}\right\|+L_{1}}{\Gamma_{q}(\alpha)} \int_{a}^{t}(t-q s)_{q}^{\alpha-1}\|z(s)\| \nabla_{q} s .
\end{aligned}
$$

By applying the result of Corollary 1 , we have

$$
\|z(t)\| \leq 0 \cdot{ }_{q} E_{\alpha}\left[\left(\left\|A_{0}\right\|+L_{1}\right) \Gamma_{q}(\alpha), t\right],
$$

which implies that $x(t)=y(t)$ for $t \in \mathbb{I}_{\tau^{-1}}$.

For $t \in\left[\tau^{-1} a, \infty\right)_{q}$, we get

$$
\begin{aligned}
z(t)= & \frac{1}{\Gamma_{q}(\alpha)} \int_{a}^{t}(t-q s)_{q}^{\alpha-1}\left[A_{0} z(s)+f(s, x(s), x(\tau s))-f(s, y(s), y(\tau s))\right] \nabla_{q} s \\
& +\frac{1}{\Gamma_{q}(\alpha)} \sum_{a}^{t}(t-q s)_{q}^{\alpha-1} A_{1} z(\tau s) \nabla_{q} s .
\end{aligned}
$$

It follows that

$$
\begin{aligned}
\|z(t)\| \leq & \frac{1}{\Gamma_{q}(\alpha)} \int_{a}^{t}(t-q s)_{q}^{\alpha-1}\left[\left\|A_{0}\right\|\|z(s)\|+\|f(s, x(s), x(\tau s))-f(s, y(s), y(\tau s))\|\right] \nabla_{q} s \\
& +\frac{\left\|A_{1}\right\|}{\Gamma_{q}(\alpha)} \int_{a}^{t}(t-q s)_{q}^{\alpha-1}\|z(\tau s)\| \nabla_{q} s \\
\leq & \frac{\left\|A_{0}\right\|+L_{1}}{\Gamma_{q}(\alpha)} \int_{a}^{t}(t-q s)_{q}^{\alpha-1}\|z(s)\| \nabla_{q} s+\frac{\left\|A_{1}\right\|+L_{1}}{\Gamma_{q}(\alpha)} \int_{a}^{t}(t-q s)_{q}^{\alpha-1}\|z(\tau s)\| \nabla_{q} s .
\end{aligned}
$$

Let $\bar{z}(t)=\sup _{\theta \in \mathbb{I}_{\tau}}\|z(\theta t)\|$, then we get

$$
\begin{aligned}
\bar{z}(t) & \leq \frac{\left\|A_{0}\right\|+L_{1}}{\Gamma_{q}(\alpha)} \int_{a}^{t}(t-q s)_{q}^{\alpha-1} \bar{z}(s) \nabla_{q} s+\frac{\left\|A_{1}\right\|+L_{1}}{\Gamma_{q}(\alpha)} \int_{a}^{t}(t-q s)_{q}^{\alpha-1} \bar{z}(s) \nabla_{q} s \\
& \leq \frac{\left\|A_{0}\right\|+\left\|A_{1}\right\|+2 L_{1}}{\Gamma_{q}(\alpha)} \int_{a}^{t}(t-q s)_{q}^{\alpha-1} \bar{z}(s) \nabla_{q} s .
\end{aligned}
$$


By applying the result of Corollary 1, we obtain

$$
\|z(t)\| \leq \bar{z}(t) \leq 0 \cdot{ }_{q} E_{\alpha}\left[\left(\left\|A_{0}\right\|+\left\|A_{0}\right\|+2 L_{1}\right) \Gamma_{q}(\alpha), t\right] .
$$

Hence, we end up with $x(t)=y(t)$ for $t \in \mathbb{T}_{\tau a}=[\tau a, \infty)_{q}$.

In the following theorem, we provide an estimate for the solution of system (19).

Theorem 6 Let condition (H.2) hold. Then the following estimate for the solution $x(t)$ of system (19) is valid:

$$
\|x(t)\| \leq\left[\|\varphi\|+\frac{L_{2}+\|\varphi\|\left(\left\|A_{0}\right\|+\left\|A_{1}\right\|\right)}{\Gamma_{q}(\alpha+1)}(t-a)_{q}^{\alpha}\right]{ }_{q} E_{\alpha}\left[\left(\left\|A_{0}\right\|+\left\|A_{1}\right\|\right) \Gamma_{q}(\alpha), t\right] .
$$

Proof For $t \in \mathbb{T}_{a}=[a, \infty)_{q}$, the solution of system (19) has the form

$$
x(t)=\varphi(a)+\frac{1}{\Gamma_{q}(\alpha)} \int_{a}^{t}(t-s)_{q}^{\alpha-1}\left[A_{0} x(s)+A_{1} x(\tau s)+f(s, x(s), x(\tau s))\right] \nabla_{q} s .
$$

It follows that

$$
\begin{aligned}
\|x(t)\| \leq & \|\varphi(0)\|+\frac{1}{\Gamma_{q}(\alpha)} \int_{a}^{t}(t-s)_{q}^{\alpha-1}\left\|A_{0} x(s)+A_{1} x(\tau s)+f(s, x(s), x(\tau s))\right\| \nabla_{q} s \\
\leq & \|\varphi\|+\frac{\left\|A_{0}\right\|}{\Gamma_{q}(\alpha)} \int_{a}^{t}(t-s)_{q}^{\alpha-1}\|x(s)\| \nabla_{q} s+\frac{\left\|A_{1}\right\|}{\Gamma_{q}(\alpha)} \int_{a}^{t}(t-q s)_{q}^{\alpha-1}\|x(\tau s)\| \nabla_{q} s \\
& +\frac{1}{\Gamma_{q}(\alpha)} \int_{a}^{t}(t-q s)_{q}^{\alpha-1}\|f(s, x(s), x(\tau s))\| \nabla_{q} s .
\end{aligned}
$$

By the assumption (H.2), the above inequality can be rewritten as

$$
\begin{aligned}
\|x(t)\| \leq & \|\varphi\|+\frac{\left\|A_{0}\right\|+\left\|A_{1}\right\|}{\Gamma_{q}(\alpha)} \int_{a}^{t}(t-q s)_{q}^{\alpha-1}\left[\sup _{\theta \in \mathbb{I}_{\tau}}\|x(\theta s)\|+\|\varphi\|\right] \nabla_{q} s \\
& +\frac{L_{2}}{\Gamma_{q}(\alpha)} \int_{a}^{t}(t-q s)_{q}^{\alpha-1} \nabla_{q} s \\
= & \|\varphi\|+\frac{L_{2}+\|\varphi\|\left(\left\|A_{0}\right\|+\left\|A_{1}\right\|\right)}{\Gamma_{q}(\alpha+1)}(t-a)_{q}^{\alpha} \\
& +\frac{\left\|A_{0}\right\|+\left\|A_{1}\right\|}{\Gamma_{q}(\alpha)} \int_{z}^{t}(t-q s)_{q}^{\alpha-1} \sup _{\theta \in \mathbb{I}_{\tau}}\|x(s \theta)\| \nabla_{q} s
\end{aligned}
$$

where the power rule (12) has been used. Let $v(t)=\|\varphi\|+\frac{L_{2}+\|\varphi\|\left(\left\|A_{0}\right\|+\left\|A_{1}\right\|\right)}{\Gamma_{q}(\alpha+1)}(t-a)_{q}^{\alpha}$, then $v$ is a nondecreasing function. Therefore, Corollary 1 implies that

$$
\|x(t)\| \leq \sup _{\theta \in \mathbb{I}_{\tau}}\|x(\theta s)\| \leq v(t)_{q} E_{\alpha}\left[\left(\left\|A_{0}\right\|+\left\|A_{1}\right\|\right) \Gamma_{q}(\alpha), t\right] .
$$

Hence, the solution $x$ of (19) satisfies the estimate

$$
\|x(t)\| \leq\left[\|\varphi\|+\frac{L_{2}+\|\varphi\|\left(\left\|A_{0}\right\|+\left\|A_{1}\right\|\right)}{\Gamma_{q}(\alpha+1)}(t-a)_{q}^{\alpha}\right]{ }_{q} E_{\alpha}\left[\left(\left\|A_{0}\right\|+\left\|A_{1}\right\|\right) \Gamma_{q}(\alpha), t\right] .
$$

The proof is complete. 
Example 2 Consider the nonlinear delay fractional difference equation of the form

$$
{ }^{q} C_{a}^{\frac{1}{2}} x(t)=2 x(t)+3 x(\tau t)-\sin x(t)+3 \sin x(\tau t), \quad t \in \mathbb{T}_{a}=[a, \infty)_{q},
$$

with the initial function $x(t)=\cos 2 t, t \in \mathbb{I}_{\tau}$. Clearly, equation (33) is a scalar equation and $A_{0}=2$ and $A_{1}=3$. The nonlinearity has the form $f(t, x(t), x(\tau t))=-\sin x(t)+3 \sin x(\tau t)$. Therefore, we have

$$
\begin{aligned}
& \|f(t, x(t), x(\tau t))-f(t, y(t), y(\tau t))\| \\
& \quad=\|-\sin x(t)+3 \sin x(\tau t)+\sin y(t)-3 \sin y(\tau t)\| \\
& \quad \leq 3(\|\sin x(t)-\sin y(t)\|+\|\sin x(\tau t)-\sin y(\tau t)\|) .
\end{aligned}
$$

Thus, condition (H.1) holds with $L_{1}=3$. By the consequence of Theorem 4, equation (33) has a unique solution. Moreover,

$$
\|f(t, x(t), x(\tau t))\|=\|-\sin x(t)+3 \sin x(\tau t)\| \leq 4,
$$

which implies that condition (H.2) is satisfied with $L_{2}=4$. By Theorem 6, the solution has the estimate

$$
\|x(t)\| \leq\left[1+\frac{9}{\Gamma_{q}\left(\frac{3}{2}\right)}(t-a)_{q}^{\frac{1}{2}}\right] \sum_{k=0}^{\infty} \frac{\left(5 \Gamma_{q}\left(\frac{1}{2}\right)\right)^{k}(t-a)_{q}^{\frac{k}{2}}}{\Gamma_{q}\left(\frac{k}{2}+1\right)}
$$

Remark 2 The following features can be concluded:

1. The delay term in system (19) can be considered as a function $\tau: \mathbb{T}_{q} \rightarrow[a-\tau, \infty) \subset \mathbb{R}$ so that the solution will be defined on the interval $[a-\tau, \infty)$. In this article our delay function acts from $[a, \infty)_{q}$ to $[\tau a, \infty)_{q} \subset \mathbb{T}_{q}$.

2. Solving equation (33) is not an easy task. However, getting a bound for the solution could be considered as a substantial step forward.

3. Clearly, equation (33) cannot be dealt with using the results of Theorem 1 and Theorem 2. Therefore, the results of this paper are essentially new and have their own merits.

\section{Conclusion}

The Gronwall inequality has an important role in many differential and integral equations. The recent years have witnessed the appearance of an increasing number of generalized Gronwall inequalities which have been addressed to overcome difficulties encountered in differential equations. To the best of the authors' knowledge, however, there is no paper that has dealt with a generalized $q$-fractional Gronwall inequality. In this paper, we extend our previous work and establish a new generalized version of discrete $q$-fractional Gronwall inequality. The new established Gronwall inequality is designed to deal with delay $q$-fractional difference systems. Therefore, we set an initial value problem involving a nonlinear delay Caputo $q$-fractional difference system. We proved the uniqueness and found an estimate for the solutions of this problem. 


\section{Competing interests}

The authors declare that they have no competing interests.

\section{Authors' contributions}

All authors contributed equally to the writing of this paper. All authors read and approved the final manuscript.

\section{Author details}

'Department of Mathematics and Physical Sciences, Prince Sultan University P. O. Box 66833, Riyadh, 11586, Saudi Arabia.

${ }^{2}$ Department of Mathematics, Çankaya University, Balgat, Ankara 06530, Turkey. ${ }^{3}$ Institute of Space Sciences, Magurele, Romania.

\section{Received: 25 August 2016 Accepted: 20 September 2016 Published online: 29 September 2016}

\section{References}

1. Finkelstein, R, Marcus, E: Transformation theory of the q-oscillator. J. Math. Phys. 36(6), 2652-2672 (1995)

2. Finkelstein, RJ: The $q$-Coulomb problem. J. Math. Phys. 37(6), 2628-2636 (1996)

3. Floreanini, R, Vinet, L: Automorphisms of the $q$-oscillator algebra and basic orthogonal polynomials. Phys. Lett. A 180(6), 393-401 (1993)

4. Floreanini, R, Vinet, L: Symmetries of the q-difference heat equation. Lett. Math. Phys. 32(1), 37-44 (1994)

5. Floreanini, R, Vinet, L: Quantum symmetries of q-difference equations. J. Math. Phys. 36(6), 3134-3156 (1995)

6. Freund, $\mathrm{PGO}$, Zabrodin, AV: The spectral problem for the $q$-Knizhnik-Zamolodchikov equation and continuous q-Jacobi polynomials. Commun. Math. Phys. 173(1), 17-42 (1995)

7. Marin, M: On existence and uniqueness in thermoelasticity of micropolar bodies. C. R. Acad. Sci. Paris, Ser. II 321(12), 475-480 (1995)

8. Marin, M, Marinescu, C: Thermoelasticity of initially stressed bodies, asymptotic equipartition of energies. Int. J. Eng. Sci. 36(1), 73-86 (1998)

9. Han, G-N, Zeng, J: On a q-sequence that generalizes the median Genocchi numbers. Ann. Sci. Math. Qué. 23(1), 63-72 (1999)

10. Marin, M: Lagrange identity method for microstretch thermoelastic materials. J. Math. Anal. Appl. 363(1), 275-286 (2010)

11. Ernst, T: A Comprehensive Treatment of $q$-Calculus. Birkhäuser, Basel (2012)

12. Samko, SG, Kilbas, AA, Marichev, Ol: Fractional Integrals and Derivatives: Theory and Applications. Gordon \& Breach, Yverdon (1993)

13. Podlubny, I: Fractional Differential Equations. Mathematics in Science and Engineering, vol. 198. Academic Press, San Diego (1999)

14. Kilbas, AA, Srivastava, HM, Trujillo, JJ: Theory and Applications of Fractional Differential Equations. North-Holland Mathematics Studies, vol. 204. Elsevier, Amsterdam (2006)

15. Jarad, F, Abdeljawad, T, Baleanu, D: Stability of q-fractional non-autonomous systems. Nonlinear Anal., Real World Appl. 14(1), 780-784 (2013)

16. Abdeljawad, T, Jarad, F, Baleanu, D: A semigroup-like property for discrete Mittag-Leffler functions. Adv. Differ. Equ. 2012, Article ID 72 (2012)

17. Bastos, NRO, Ferreira, RAC, Torres, DFM: Necessary optimality conditions for fractional difference problems of the calculus of variations. Discrete Contin. Dyn. Syst., Ser. A 29(2), 417-437 (2011)

18. Bastos, NRO, Ferreira, RAC, Torres, DFM: Discrete time variational problems. Signal Process. 91(3), $513-524$ (2011)

19. Atici, FM, Şengül, S: Modeling with fractional difference equations. J. Math. Anal. Appl. 369(1), 1-9 (2010)

20. Atici, FM, Eloe, PW: A transform method in discrete fractional calculus. Int. J. Difference Equ. 2(2), 165-176 (2007)

21. Atici, FM, Eloe, PW: Initial value problems in discrete fractional calculus. Proc. Am. Math. Soc. 137(3), 981-989 (2009)

22. Goodrich, CS: Continuity of solutions to discrete fractional initial value problems. Comput. Math. Appl. 59(11), 3489-3499 (2010)

23. Goodrich, CS: Solutions to a discrete right-focal fractional boundary value problem. Int. J. Difference Equ. 5(2), 195-216 (2010)

24. Anastassiou, GA: Nabla discrete fractional calculus and nabla inequalities. Math. Comput. Model. 51(5-6), 562-571 (2010)

25. Cheng, J-F, Chu, Y-M: Fractional difference equations with real variable. Abstr. Appl. Anal. 2012, Article ID 918529 (2012)

26. Cheng, JF, Wu, GC: Solutions of fractional difference equations of order (2, q). Acta Math. Sin. 55(3), $469-480$ (2012)

27. Annaby, MH, Mansour, ZS: q-fractional Calculus and Equations. Lecture Notes in Mathematics, vol. 2056. Springer, Heidelberg (2012)

28. Atici, FM, Eloe, PW: Fractional q-calculus on a time scale. J. Nonlinear Math. Phys. 14(3), 333-344 (2007)

29. Rajkovic, PM, Marinkovi, SD, Stankovi, MS: Fractional integrals and derivatives in q-calculus. Appl. Anal. Discrete Math. 1(1), 311-323 (2007)

30. Mansour, ZSI: Linear sequential q-difference equations of fractional order. Fract. Calc. Appl. Anal. 12(2), 159-178 (2009)

31. Zhao, Y, Chen, H, Zhang, Q: Existence results for fractional $q$-difference equations with nonlocal $q$-integral boundary conditions. Adv. Differ. Equ. 2013, Article ID 48 (2013)

32. Abdeljawad, T, Baleanu, D: Caputo $q$-fractional initial value problems and a $q$-analogue Mittag-Leffler function. Commun. Nonlinear Sci. Numer. Simul. 16(12), 4682-4688 (2011)

33. Abdeljawad, T, Benli, B, Baleanu, D: A generalized q-Mittag-Leffler function by Caputo fractional linear equations. Abstr. Appl. Anal. 2012, Article ID 546062 (2012)

34. Ye, H, Gao, J, Ding, Y: A generalized Gronwall inequality and its application to a fractional differential equation. J. Math. Anal. Appl. 328(2), 1075-1081 (2007)

35. Ma, Q-H, Pecari, J: Some new explicit bounds for weakly singular integral inequalities with applications to fractional differential and integral equations. J. Math. Anal. Appl. 341(2), 894-905 (2008) 
36. Furati, KM, Tatar, N-E: Inequalities for fractional differential equations. Math. Inequal. Appl. 12(2), 279-293 (2009)

37. Kong, Q-X, Ding, X-L: A new fractional integral inequality with singularity and its application. Abstr. Appl. Anal. 2012, Article ID 937908 (2012)

38. Atici, FM, Eloe, PW: Gronwall's inequality on discrete fractional calculus. Comput. Math. Appl. 64(10), 3193-3200 (2012)

39. Lin, S-Y: Generalized Gronwall inequalities and their applications to fractional differential equations. J. Inequal. Appl. 2013, Article ID 549 (2013)

40. Abdeljawad, T, Alzabut, J: The $q$-fractional analogue for Gronwall-type inequality. J. Funct. Spaces Appl. 2013, Article ID 543839 (2013)

Submit your manuscript to a SpringerOpen ${ }^{\odot}$ journal and benefit from:

$\rightarrow$ Convenient online submission

Rigorous peer review

- Immediate publication on acceptance

- Open access: articles freely available online

- High visibility within the field

- Retaining the copyright to your article 